\title{
Fabrication of multiple parallel suspended-core optical fibers by sheet- stacking
}

\author{
Jindan Shi, Xian Feng *, Zhenggang Lian, Nicholas White, Wei H. Loh, Francesco Poletti, \\ Peter Horak \\ Optoelectronics Research Centre, University of Southampton, Southampton, SO17 1BJ, UK \\ *Corresponding author: xif@ orc.soton.ac.uk
}

Keywords: non-silica glass fiber; microstructured optical fiber; fiber design and fabrication

\begin{abstract}
We demonstrate the fabrication of a novel type of optical fibers with multiple parallel airsuspended cores by the sheet-stacking method. Using this technique we have constructed optical fibers with up to 10 parallel micron-size suspended cores. No extra scattering loss from the fabrication process was observed in a fabricated dual air-suspended core fiber. The sheetstacking method opens the way towards using a wide range of optical glasses for manufacturing multiple parallel suspended-core specialty optical fibers with novel optical functionalities such as dispersion tunability. Fusion splicing has also been successfully used to connect such a multiple core fiber with a conventional silica fiber.
\end{abstract}

\section{Introduction}

Multi-core optical fiber technology is an attractive and promising approach to enhance the functionalities and capacities of the traditional single core optical fiber. One example is the recent progress of using multicore single-mode fibers for largely enhancing the transmission capacity of the long-haul fiber optical telecommunication network [1,2].

Multicore optical fiber technology is also a powerful way for achieving novel optical components such as broadband directional couplers [3], narrow-band fiber filters [4], variable fiber attenuators [5], two-dimensional bending sensors [6], fiber lasers and amplifiers [7,8], and dispersion compensators [9]. For these applications, due to the rigid core/cladding physical structure, the fiber geometry is static, i.e., relative core-to-core movement is not possible. However, if the core-to-core separation can be modified in a well-controlled manner, this additional degree of freedom will further enhance the functionality and capability of multicore optical fiber technology in the areas of optical sensing, optical switching and optical processing. This motivates our target to develop novel multicore optical fibers with movable cores.

In a conventional all-solid optical fiber, core and cladding are composed of different glasses. With the invention of holey fibers (HFs) [10] it became possible to fabricate single-material optical fibers with air-filled microstructured cladding. Most HFs demonstrated to date have quite short and thick glass bridges between the air holes in the cladding. Thus the cores of these HFs are static, just as in conventional solid optical fibers. An exception is a HF exhibiting a micron- 
size core attached to a long glass membrane with submicron thickness. For this type of airsuspended-core (ASC) HF the light is guided along the glass core, which is surrounded by the air cladding, and thus optically the core is effectively suspended in air. Because of the long and thin supporting membrane, the core can be physically displaced from its stationary position by an appropriate external force. By adding a second air-suspended core parallel to the first one with a small distance on the wavelength scale, any relative movement between the two cores can be observed optically due to the light coupling between the two neighboring cores.

Such a type of multiple parallel ASC fibers with two cores has already been demonstrated $[11,12]$. When an external force, such as air pressure [12], electrostatic actuation [13] or even optical force [14-16], is applied to one of the cores, the light coupling between the neighboring cores can be modified in a well-controlled manner. In order to allow the mechanical actuation of the cores by weak external forces and also to ensure low confinement loss of the cores, the supporting glass membranes are required to be longer than $10 \mu \mathrm{m}$ and thinner than $200 \mathrm{~nm}$. However, these requirements present a significant challenge for the fiber fabrication.

So far, two methods have been employed to fabricate such a type of novel optical fibers: (i) capillary stacking and (ii) glass extrusion.

In the former case, silica glass was adopted as the fiber host. Three major capillaries and four assisting capillaries were stacked inside a jacket tube (as shown in Fig.1(a)) and high air pressure was applied into these three major capillaries so that two thin and long membranes were finally formed in the fiber [16,17]. But it should be noted that there was no core formed in the fiber and the transmission loss at $1550 \mathrm{~nm}$ was therefore measured to be $35 \mathrm{~dB} / \mathrm{m}$, which is extremely high for silica based index-guided holey fibers. As described in Ref. [17], attempts to attach cores to the capillaries in the stacking were made but a large offset was observed between the two formed cores and the core size was still as large as several microns in diameter. This is because the high pressure can expand the capillary and make the membrane as thin as a few hundred nanometers and as long as tens of micrometers [18], but due to mass preservation the core size will approximately follow the reduction of the outer fiber diameter from that of the preform.

In the second fabrication method, glass extrusion [11,12], a non-silica glass (commercial lead silicate glass, Schott F2) was chosen as the fiber host material. The dual-core fiber preform was made by extrusion under a temperature around $600^{\circ} \mathrm{C}$. The extrusion method is a well-developed method for making non-silica glass (or so-called soft glass) HF [19] above the glass softening temperature, which is typically below $800{ }^{\circ} \mathrm{C}$. It is very powerful in directly making microstructured preforms with glass features even as small as $7 \mu \mathrm{m}$ [20]. But due to the lack of suitable die materials capable of working at temperatures above $1000{ }^{\circ} \mathrm{C}$ and at high pressures of $0.1-10 \mathrm{kN} / \mathrm{cm}^{2}$, the glass extrusion technology has not shown any success in making preforms using glasses with high softening temperature such as silica.

Therefore, it is necessary to develop a new approach for the fabrication of multiple parallel ASC fibers using a wide range of materials covering both silica and non-silica glasses.

Here we demonstrate an interesting alternative to produce the geometry of multiple parallel ASC optical fibers: We employ a sheet stacking method where a unit element comprising a glass core sitting on a thin glass sheet is periodically repeated. In the simplest case, a single-ASC fiber can be drawn from a preform consisting of a core rod, a thin sheet and a surrounding thick 
walled jacket tube [10], as illustrated in Fig. 2 (a). Fibers with multiple parallel suspended cores can be built on this platform, as shown in Fig. 2 (b)\&(c). The obvious advantages of this approach over the extrusion method are that (i) no metal extrusion die needs to be fabricated, (ii) the materials for the core and the membrane can be different from each other and (iii) different materials can be used for different cores in the same fiber, as long as all materials employed are thermally compatible during the fabrication [21].

(a)

\section{Capillary stacking}

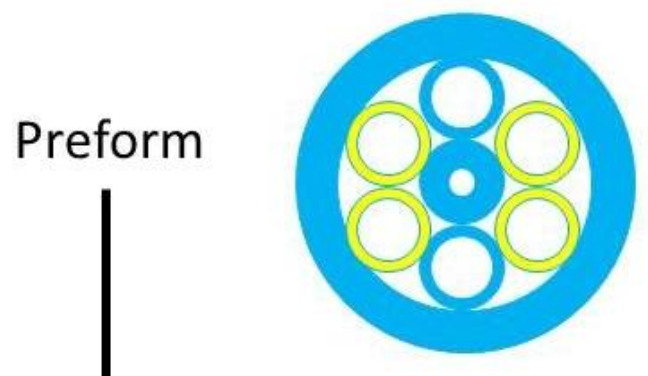

Cane

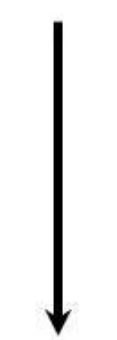

Fiber
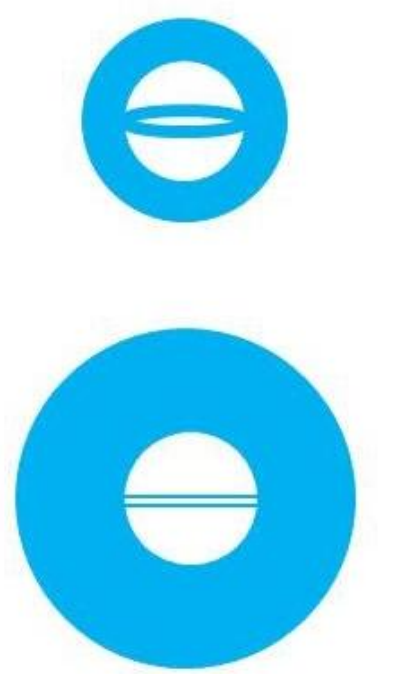

(b)

\section{Extrusion}
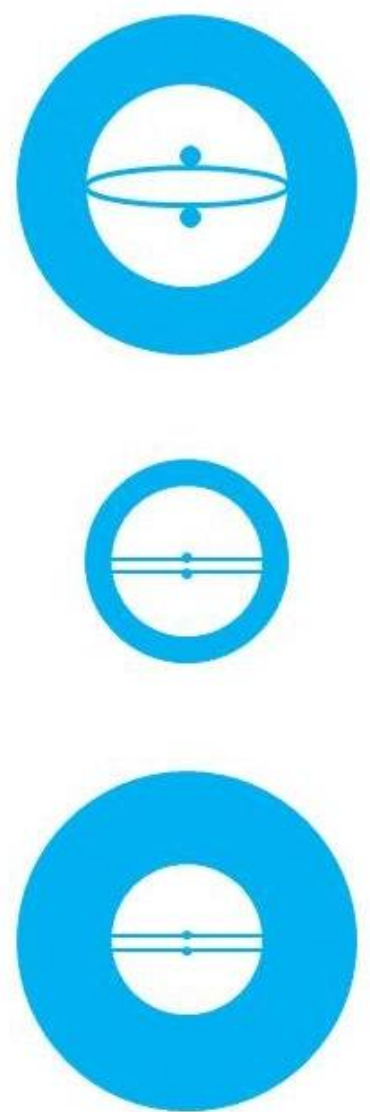

Fig. 1. Schematic diagrams of (a) the capillary-stacking method to fabricate dual-membrane fiber and (b) the extrusion method to make dual-ASC HF. Note that in the case of (a), during the caning procedure from the preform to the cane, high gas pressure was applied to the central three major capillaries (in blue). The four side assisting capillaries (in yellow) were collapsed after caning. For both cases, at the last step of fiber drawing, the cane was inserted into a jacket tube and this ensemble was drawn into the dual-ASC (or dual-nanoweb) fiber.

( a )

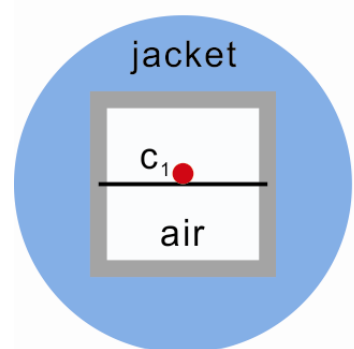

( b )

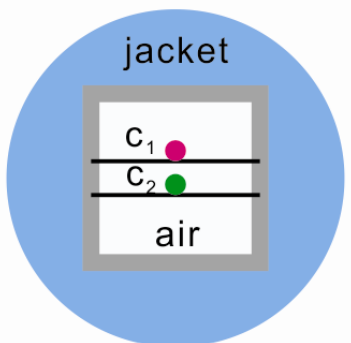

(c)

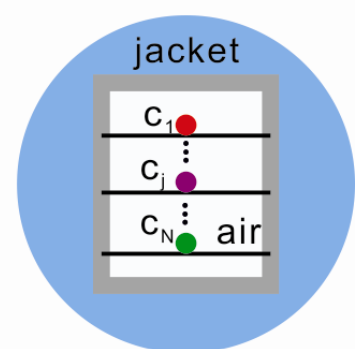

Fig. 2. (a) Schematic diagrams of the sheet-stacking method to build preforms for (a) single-, (b) dual- and (c) NASC fibers. In our experiments, supporting membranes (black), fiber cores $\left(C_{1}\right.$ to $\left.C_{N}\right)$, outer walls (gray) and jacket tube (blue) are made of Schott D263T, N-BK7, N-BK7 and LLF1 glasses, respectively. 
In this work, two thermally-compatible commercial borosilicate glasses have been used for the fiber cores and membranes. A dual-ASC fiber and a 10-ASC fiber with micron-size cores and long and thin membranes have been fabricated, indicating that the sheet stacking method is a scalable approach to fabricate multiple cores. The loss of the dual-ASC fiber has been measured to be the same as the bulk loss of the original core glass, showing that the extra loss introduced by this approach is negligible. Numerical simulations show that the dual-ASC fiber can be used as a broadband dispersion tuning device by adjusting the core-to-core distance. In addition, fusion splicing has been successfully used for connecting the dual-ASC fiber with a commercial silica fiber.

\section{Fabrication of multiple parallel ASC holey fibers}

\subsection{Preform preparation and fiber drawing of dual-ASC fiber}

The sheet stacking method was inspired by the procedure of Kaiser used in the early 1970s to make the first single-material silica HF, i.e., a single-ASC HF [10, 22]. In that work, the ensemble of the preform included a silica core rod with a diameter up to $1.5 \mathrm{~mm}$, a polished silica supporting sheet with a thickness of $100 \mu \mathrm{m}$ and a width of $6.5 \mathrm{~mm}$, and a silica jacket tube with an inner diameter (ID) of $6.5 \mathrm{~mm}$ and an outer diameter (OD) of $10 \mathrm{~mm}$. The core rod was either centered in the jacket tube by a capillary tube or attached to the top end of the supporting sheet with high temperature cement. Because the reduction ratio from the preform to the fiber was approximately 100 to 1 , the preform was directly drawn into fiber with diameters of 100-200 $\mu \mathrm{m}[22]$.

As described above, the multiple parallel ASC HF requires center-to-center distance between the neighboring cores in the fiber of wavelength scale in order to have strong light coupling between the cores. The reduction ratio from the preform to the fiber should therefore be approximately 1000 to 1 , if the starting core rod of the preform is $1 \mathrm{~mm}$ in diameter. This is impractical, and an intermediate caning process is thus necessary before the final fiber drawing. This step was not included in Kaiser's works for making a single-ASC HF [22]. As we will show in the following, the caning process is crucial for fabricating multiple parallel ASC HF rather than a single-ASC HF, because an offset of the neighboring cores will occur if the fabrication conditions are inappropriate.

We chose commercial Schott N-BK7 glass and D263T glass as the materials for the cores and sheets, respectively. These glasses are both borosilicate glasses but with slightly different thermal properties [23]. The concept of the construction of the preform with $\mathrm{N}$ cores $(\mathrm{N}=2$ and 10 ) is schematically illustrated in Fig. 2 (b\&c). The outer walls of the preform were constructed using four pieces of matted N-BK7 plates, each with $2 \mathrm{~mm}$ thickness. N slots of $1.0 \mathrm{~mm}$ depth and $200 \mu \mathrm{m}$ width were machined on each vertical wall using a commercial dicing saw (Isomet 5000 Linear Precision Saw). The gap between the neighboring slots was precisely controlled between 1.3-1.5 mm by the digital micrometer gauge on the dicing saw. Commercial borosilicate glass sheets (Schott D263T) with a thickness of $100 \mu \mathrm{m}$ and a width of $22 \mathrm{~mm}$ were employed in the stacked preform. Each sheet was horizontally slipped into the pair of symmetric slots on the 
vertical walls (as illustrated in Fig. 2 (b\&c)). An N-BK7 glass core was then placed on the top of each sheet. The cores with a rectangular cross section of $\sim 720 \times 700 \mu \mathrm{m}$ were elongated from a sawed N-BK7 rod. The cores were carefully aligned along the vertical central line of the preform. This stacked preform was heated in a furnace at $600-700{ }^{\circ} \mathrm{C}$ for $0.5-2$ hours to fuse all the pieces of the preform together, and then annealed at the glass transition temperature $\mathrm{T}_{\mathrm{g}}=557$ ${ }^{\circ} \mathrm{C}$ for 2 hours. Thin graphite sheets were placed below the D263T sheets to prevent them from sagging during the heating, and then removed prior to the caning process. A cane with multiple parallel cores was elongated from the fused preform. The cane was then inserted into an extruded Schott LLF1 glass jacket tube with an outer diameter (OD) of $10 \mathrm{~mm}$ and an inner diameter (ID) marginally bigger than the cane. The preform ensemble was finally drawn into fiber.

Fig. 3 illustrates the viscosity curves of the three glasses used for cores, sheets and jacket tube. It shows that the thermal mismatch between these glasses is compatible for the fabrication procedure described above. The fiber drawing temperature in our experiment was controlled between $800-850{ }^{\circ} \mathrm{C}$.

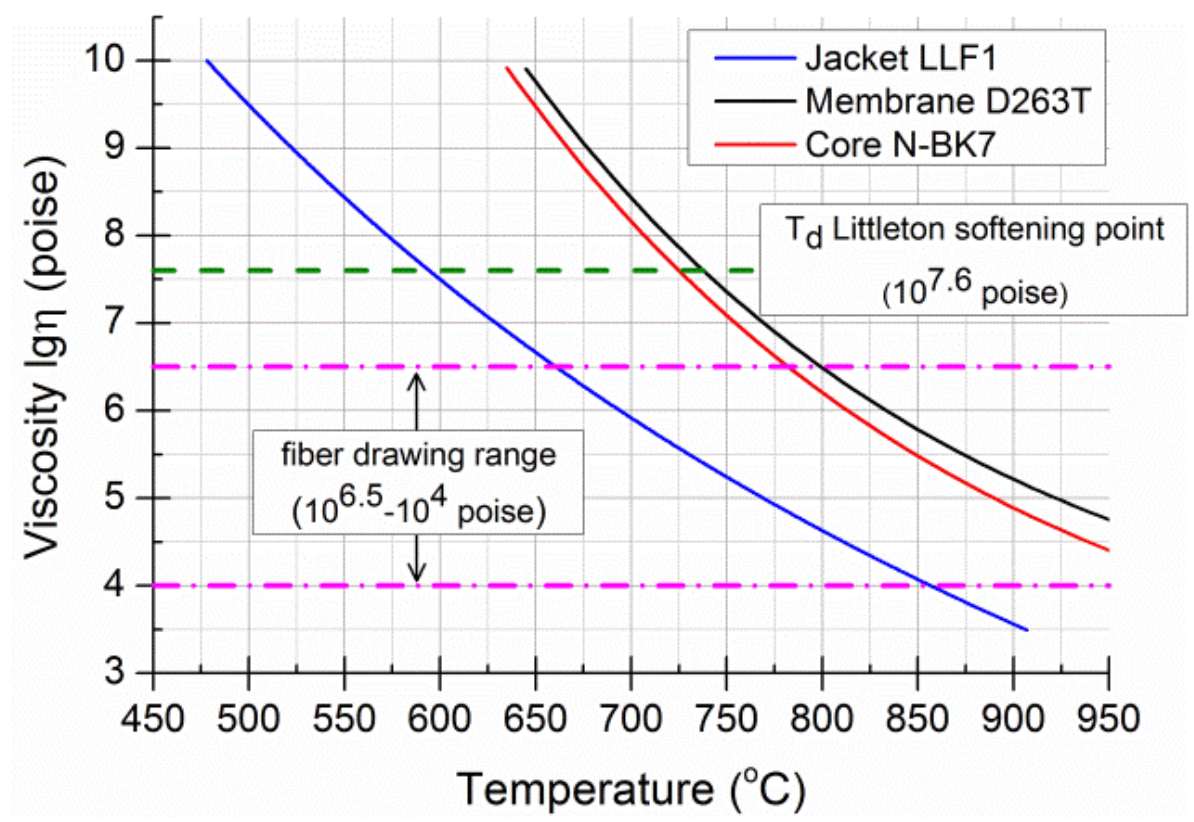

Fig. 3. Viscosity curves of LLF1 (for jacket), N-BK7 (for cores) and D263T (for membranes) glasses.

Fig. 4 (a) shows a photograph of the annealed dual-ASC stacked preform. Fig. 4 (b) gives a scanning electron microscope (SEM) photograph of the cane with a diagonal length of $2.1 \mathrm{~mm}$. The cane was then inserted into an extruded LLF1 jacket tube with $10 \mathrm{~mm}$ OD and $2.2 \mathrm{~mm}$ ID. This preform ensemble was then drawn into the dual-ASC fiber. The yield of the fiber drawing was 100 meters. Fig. 4 (c) shows an SEM image of the dual-ASC fiber with an OD of $160 \mu \mathrm{m}$ and two cores with dimensions of $0.78 \times 2.7 \mu \mathrm{m}$. The center-to-center distance between the cores is $2.4 \mu \mathrm{m}$. The two supporting membranes have a uniform thickness in the range between 137 $\mathrm{nm}$ and $149 \mathrm{~nm}$ and a length of $39.4 \mu \mathrm{m}$. Such long thin membranes were achieved by applying positive pressure to the air holes on the cane during fiber drawing. Because the LLF1 glass has lower viscosity than the N-BK7 glass, the jacket is more liquideous than the cane and it therefore keeps exerting pressure on the central cane. During the fiber drawing, an air flow was forced under pressure into the three holes of the cane to expand the holey structure. Because the central 
hole is smaller than the two side ones, air flowing into this hole experiences higher friction and the effective pressure in the central hole was thus weaker than that in the two larger side holes. The observed curvature of the membranes was the outcome of the combination of these two effects.
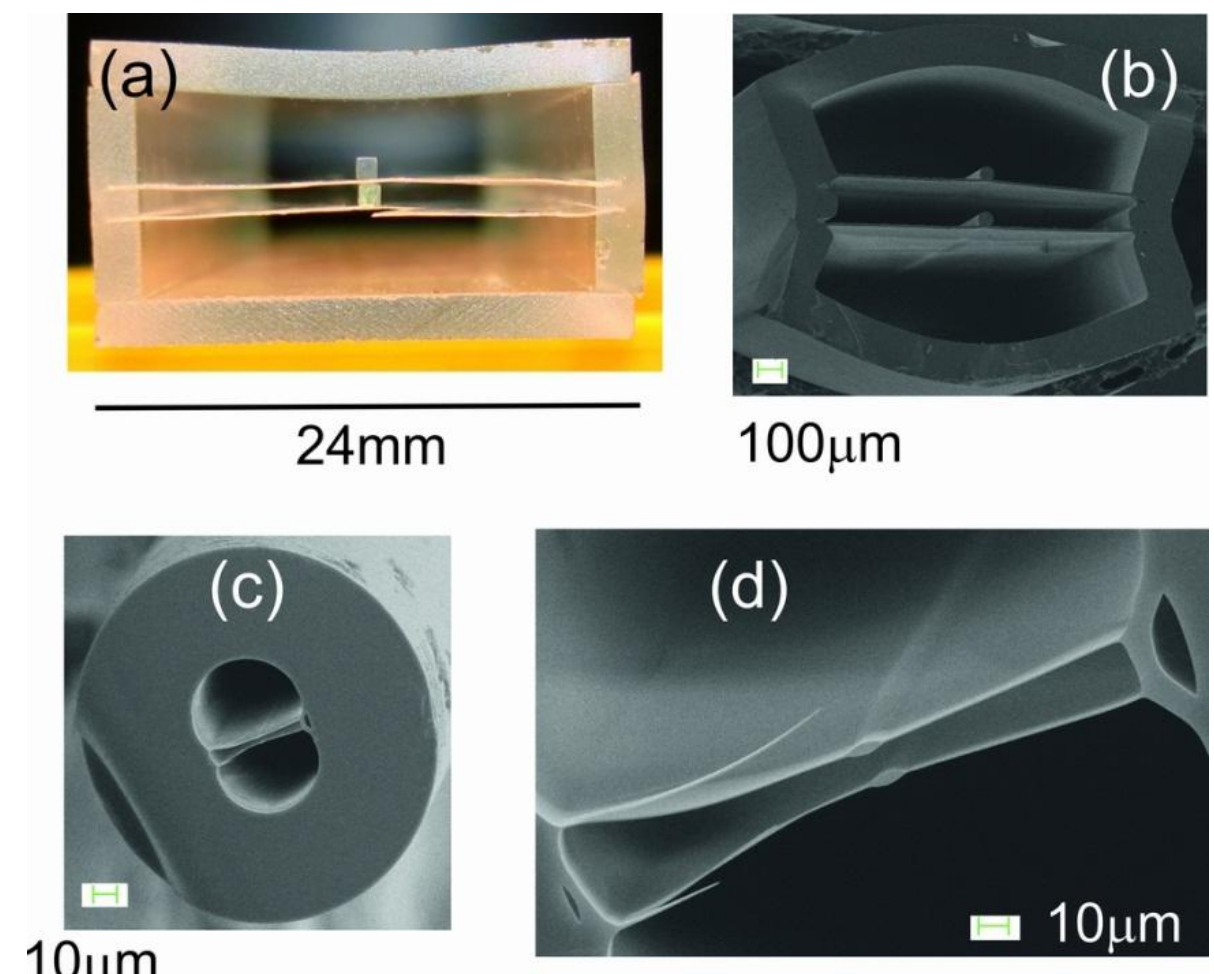

Fig. 4. (a) Photograph of a stacked dual-ASC preform; SEM photos of (b) dual-ASC cane, (c) dual-ASC fiber and (d) central part of dual-ASC fiber.

\subsection{Variation of membrane thickness in dual-ASC fiber}

For precise control of the mechanical and optical properties of the fiber, the thickness uniformity of the supporting membranes is important. We thus analyzed fibers fabricated by the different methods described above.

Fig.5 compares the SEM photographs of the dual-ASC HF from sheet-stacking and extrusion. Table 1 summarizes the thickness and the thickness variation of the membranes of the dual-ASC (or dual-nanoweb) fiber made by sheet stacking, capillary stacking, and extrusion. It is seen that the thickness of the HF made from the former method is very uniform and the thickness variation is $\sim 5 \mathrm{~nm}$, which is the resolution of the SEM used for imaging dielectric materials like glasses. The relative standard deviation of the thickness in the HF made by sheet-stacking approach is even twice as low as the one in the HF made from capillary-stacking method [18]. Note that because of lack of the information of the thickness variation for the dual-nanoweb fiber [16,17], the values listed in Table 1 were from a single-nanoweb fiber made by capillary stacking [18]. The dual-ASC fiber from the extrusion method shows the worst thickness uniformity of the membrane because of (i) the variation of the slot width $(300-400 \mu \mathrm{m})$ on the metal die used for extrusion and (ii) the temperature gradient of the glass flow filling into the slot during the extrusion. 


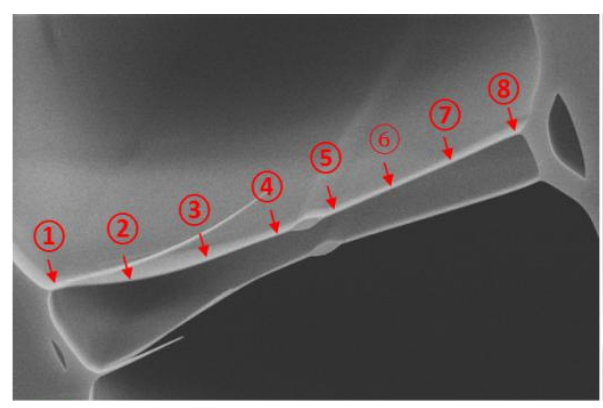

$2 \mu \mathrm{m}$

(a)

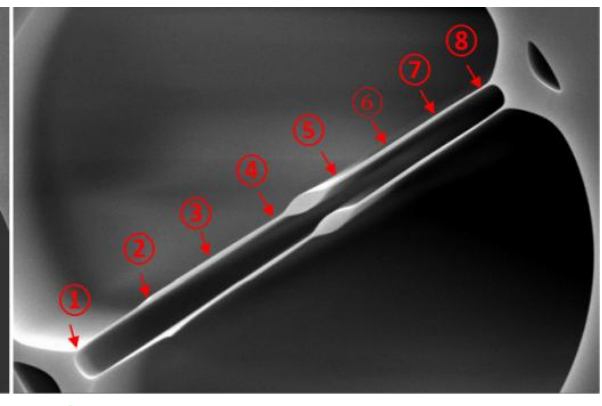

$1 \mu \mathrm{m}$

(b)

Fig. 5. SEM photographs of the dual-ASC fiber fabricated by (a) sheet stacking and (b) extrusion. The thicknesses of each membrane have been taken from eight positions on one membrane of both fibers, as shown on the photos.

Table 1 Comparison of thickness variation of membrane of dual-ASC fibers (or dual-nanoweb fiber) made by sheet stacking, extrusion, and capillary stacking. Note that the standard deviation $\sigma$ is defined as $\sigma=\sqrt{\frac{1}{N} \sum_{1}^{N}\left(x_{i}-\bar{x}\right)^{2}}$, where $\mathrm{N}$ is the number of the data $\mathrm{x}_{\mathrm{i}}$, and the $\bar{x}$ is the mean of $\mathrm{x}_{\mathrm{i}}(\mathrm{i}=1-\mathrm{N})$, respectively.

\begin{tabular}{|l|r|r|r|}
\hline Method & $\begin{array}{r}\text { Average } \\
\text { membrane } \\
\text { thickness (nm) }\end{array}$ & $\begin{array}{r}\text { Standard } \\
\text { deviation } \\
(\mathrm{nm})\end{array}$ & $\begin{array}{r}\text { Relative } \\
\text { standard } \\
\text { deviation }\end{array}$ \\
\hline Capillary stacking [18] & 610 & 40 & $6.6 \%$ \\
\hline Extrusion [12] & 210 & 90 & $43 \%$ \\
\hline Sheeting stacking (this work) & 140 & 5 & $3.6 \%$ \\
\hline
\end{tabular}

\subsection{Scalability of the sheet-stacking method}

The possibility of introducing more than two cores is promising for potential applications such as beam steering [24] and optical sensing with multiple functionalities. A stacked 10-ASC preform is seen in Fig. 6 (a). Fig. 6 (b) shows an SEM photograph of the cane with a diagonal length of $3.2 \mathrm{~mm}$. The cane was then inserted into an extruded LLF1 jacket tube with $10 \mathrm{~mm}$ OD and $3.5 \mathrm{~mm}$ ID. Using the same procedure as described above, the very first 10-ASC optical fiber has been successfully fabricated with a yield of $40 \mathrm{~m}$. No positive pressure was applied during the fiber drawing. SEM photographs of the 10-ASC fiber are shown in Fig. 6 (c) \& (d). The fiber has an OD of $160 \mu \mathrm{m}$ and 10 parallel suspended cores with diameters between 1.05 $1.45 \mu \mathrm{m}$. The variation of the core diameters is due to the non-uniformity of the starting core rods in the stacked preform. The center-to-center distances between the neighboring cores are between 3.3 and $4.4 \mu \mathrm{m}$, due to the varied curvatures of the membranes. The thickness of the membranes is between 210 and $340 \mathrm{~nm}$.

The large variation of the membrane thicknesses in the 10-ASC fiber is due to the different curvature of the membranes resulting in a different arc length for each membrane, which is in the range of $21.7-27.4 \mu \mathrm{m}$. For most applications all the membranes should be ideally parallel to each other and the cores should be aligned in a line. We expect that all these deviations from the ideal design (as shown in Fig. 2 (c)) will be rectified in the future by precisely controlling and optimizing the fabrication parameters, such as preform dimensions, drawing temperature, pressure selectively applied into the air holes and so on.

In addition, based on the deviation of the above 10-ASC fiber from the ideal 10 parallel ASC fiber, it can be seen that fabricating a multiple parallel ASC fiber is not just a straightforward 
extension of the single-core fiber demonstrated by Kaiser [10,22]. The latter was a one-step fabrication from the preform to the fiber, because the fiber core was relatively large (diameter $\gtrsim 5 \mu \mathrm{m}$ ). To achieve core diameters close to $1 \mu \mathrm{m}$ in the multiple parallel ASC fiber, introducing the intermediate caning process was needed. The structural imperfections of the fiber, such as the misalignment of the core array, the varied curvature of the membranes, and the thickness variation of the membranes, tend to occur after the multiple thermal processes, but we are confident that all can be addressed with further development of the technique.

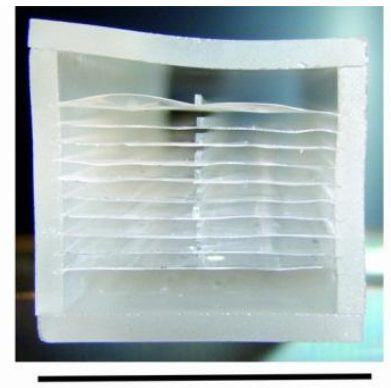

$24 \mathrm{~mm}$

(a)

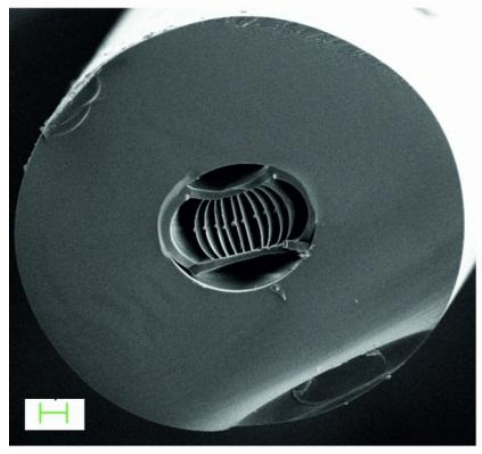

$10 \mu \mathrm{m}$

(c)

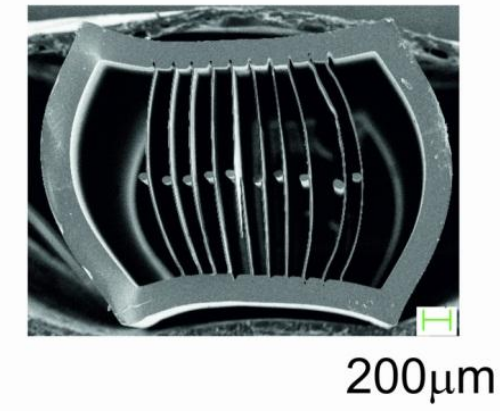

(b)

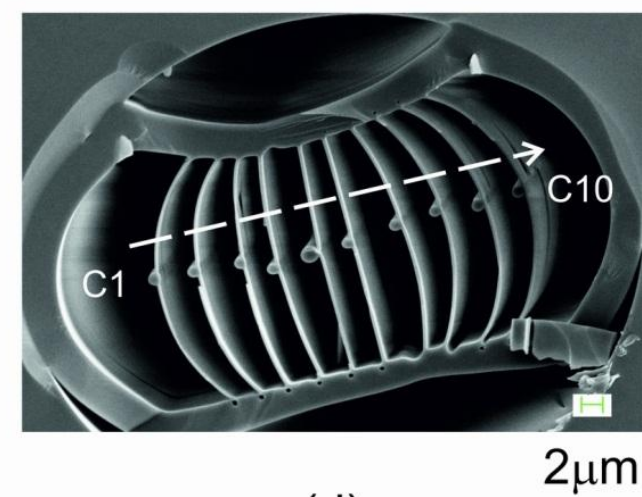

(d)

Fig. 6. (a) Photograph of a stacked 10-ASC preform; SEM photos of (b) 10-core cane, (c) 10-ASC fiber and (d) central part of 10-ASC fiber. In (d) the 10 cores have been labeled with $\mathrm{C} 1$ to $\mathrm{C} 10$ respectively.

\section{Results and discussion}

\subsection{Optical performance of fabricated dual-ASC fiber and 10-ASC fiber}

For our dual-ASC fiber with elliptical cores of $0.78 \times 2.7 \mu \mathrm{m}$ (see Fig. 4 (d)), finite-element simulations indicate that the fiber is slightly multimoded at $1550 \mathrm{~nm}$ : It supports two TE (horizontal polarization) modes and two TM (vertical polarization) modes per core. In our experiments, only the fundamental TE and TM modes have been excited. Fig. 7 (a) shows the near-field mode profile from the output end of the dual-ASC fiber, measured with a near-infrared (NIR) camera (WinCamD-UCD12, Data Ray Inc.). The intensity profiles from both cores show good agreement with a Gaussian distribution (see Fig. 7 (b)), indicating that the two cores are both effectively single-moded at $1550 \mathrm{~nm}$. 
Fig. 7 (c) shows the near-field image at the wavelength of $1550 \mathrm{~nm}$ from the 10-ASC fiber when all the cores were illuminated. An infrared camera (MicronViewer, Model 7290A from Electrophysics) was employed in the experiment. By using a high-NA focal lens and carefully adjusting the launching conditions, the light can be launched into each individual core. Fig. 7 (d) shows the near-field images at the wavelength of $1550 \mathrm{~nm}$ from the core $\mathrm{C} 1$ and core $\mathrm{C} 2$ in the 10 -ASC fiber respectively. Effectively single-moded guidance has been confirmed at $1550 \mathrm{~nm}$ in the cores of the 10-ASC fiber. It is also seen that the output intensity from the ten different cores varies because (i) the launching situation for each individual core was not exactly identical and (ii) the cleave quality of the ten cores was not equally good. How to solve these technical difficulties for such a fiber with many micron-size cores will be the subject for future research.

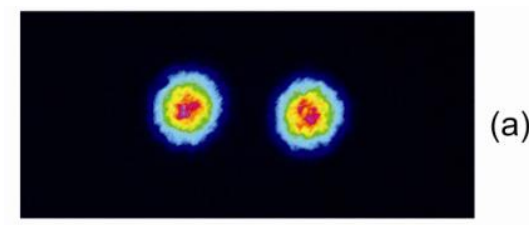

(a)

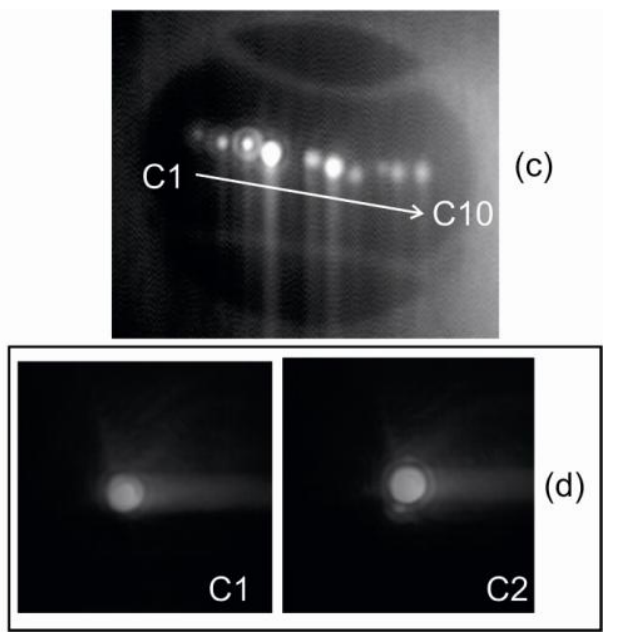

Fig. 7. (a) Observed near-field image and (b) Gaussian fitted intensity profile of the fundamental TE mode $\left(\mathrm{TE}_{00}\right)$ from the dual-ASC fiber at $1550 \mathrm{~nm}$; (c) near-field image of output of 10-ASC fiber when all the ten cores were illuminated; (d) observed near-field images from individual core C1 and core C2 of the 10-ASC fiber at $1550 \mathrm{~nm}$.

With the cut-back method the loss of the dual-ASC fiber was measured as $3.9 \pm 0.1 \mathrm{~dB} / \mathrm{m}$ at $1550 \mathrm{~nm}$ for both fundamental TE and TM modes, as seen in Fig. 8. For comparison, an N-BK7 unclad fiber with an OD of $230 \pm 5 \mu \mathrm{m}$ was also drawn from a glass rod drilled from bulk glass. The loss of this unclad fiber was measured to be $3.9 \pm 0.05 \mathrm{~dB} / \mathrm{m}$, indicating that the additional loss arising from the sheet-stacking approach itself is negligible.

For the fabricated 10-ASC fiber, the loss from the core $\mathrm{C}_{1}$ (see Fig. 6 (c)) was measured at $1550 \mathrm{~nm}$ using cutback method. With the assistance of the CCD camera, the light was launched into the core $\mathrm{C} 1$ (see Fig.7(d)). Because the center-to-center distance between core $\mathrm{C} 1$ and $\mathrm{C} 2$ is $3.6 \mu \mathrm{m}$, the light coupling between $\mathrm{C} 1$ and $\mathrm{C} 2$ is thus negligible (see Fig.7(d)). The propagation loss of the core $\mathrm{C} 1$ is measured to be $6.3 \pm 0.1 \mathrm{~dB} / \mathrm{m}$ at $1550 \mathrm{~nm}$ for both fundamental TE and TM modes, as illustrated in Fig. 8. This is higher than the losses measured in the dual-ASC fiber. Since the simulations indicate that the confinement loss of each core here should be negligible at $1550 \mathrm{~nm}$, the enhanced loss in the 10-ASC fiber might be due to (i) imperfections formed in the fabrication procedure, e.g., the contaminations of the stacked preform or the cane from the handling, and/or (ii) residual defects from the commercial glass sheets. The latter can be seen on the SEM pictures in Fig.6 (d): there are some visible micro-cracks on the membranes but they are not seen in the dual-ASC fiber in Fig. 4 (d). 


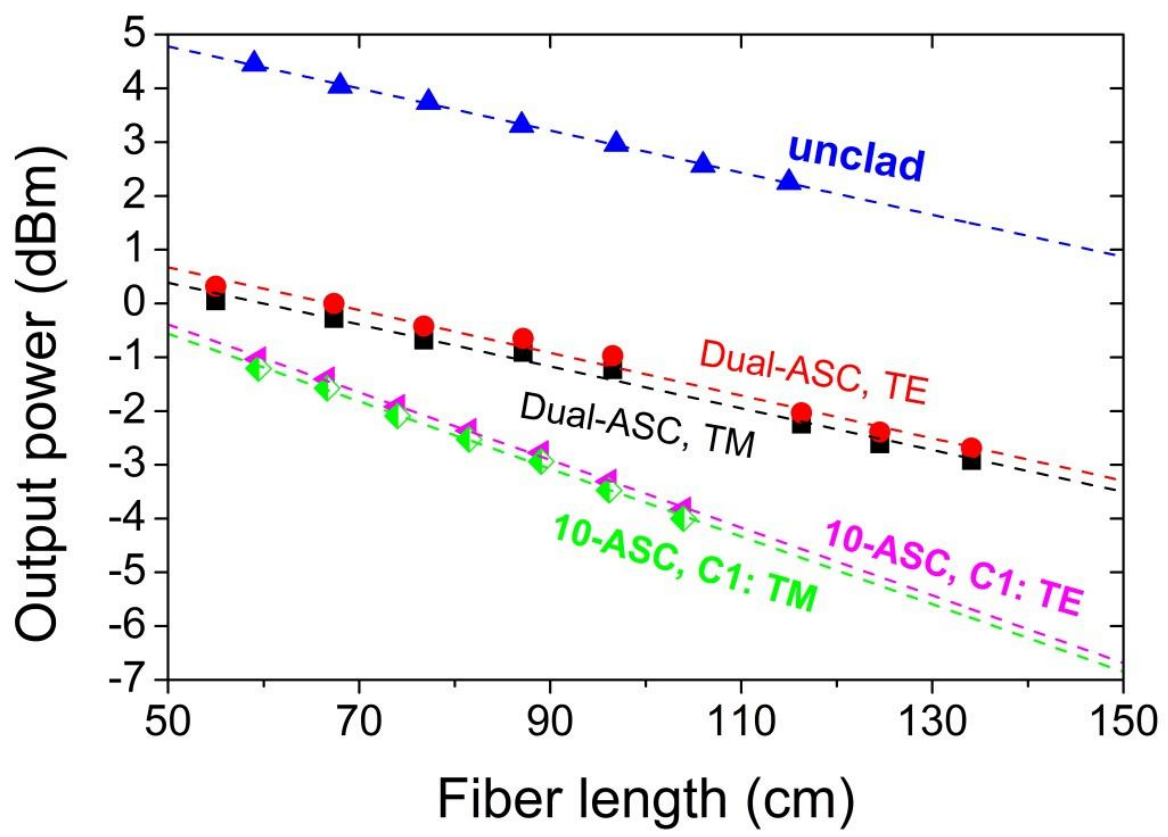

Fig. 8. (a) Comparison of measured propagation losses of fabricated unclad fiber, dual-ASC fiber and 10-ASC fiber at $1550 \mathrm{~nm}$

Table 2 compares the loss of the dual-ASC (or dual-nanoweb) holey fibers made by extrusion [12], capillary stacking [18] and sheet-stacking (this work) at $1550 \mathrm{~nm}$. It shows that only the fiber made by the sheet stacking method shows negligible extra loss from the fabrication procedures, while the fibers made by extrusion and capillary stacking both suffer from a significant increase of the loss arising from their own fabrication approaches, indicating the advantage of using the sheet-stacking method for fabricating low-loss multiple parallel ASC fiber.

Table 2 Comparison of measured loss at 1550nm of dual-ASC or dual-nanoweb fiber made by extrusion, sheet stacking and capillary stacking

\begin{tabular}{|l|c|c|}
\hline Method & $\begin{array}{c}\text { Measured } \\
\text { loss }(\mathrm{dB} / \mathrm{m})\end{array}$ & $\begin{array}{c}\text { Bulk loss } \\
(\mathrm{dB} / \mathrm{m})\end{array}$ \\
\hline Capillary stacking [18] & 35 & $<10^{-3}$ \\
\hline Extrusion [12] & 2.5 & 1.8 \\
\hline Sheeting stacking (this work) & 3.9 & 3.9 \\
\hline
\end{tabular}

\subsection{Simulation of dispersion tunability of dual-ASC fiber}

Fig.9 shows our numerical simulations on the dispersion tunability of the fabricated dual-ASC fiber when changing the center-to-center distance between the two cores. For this fiber we find that amongst the symmetric and antisymmetric fundamental TE and TM modes, the antisymmetric TE mode shows reasonably good tunability of the zero-dispersion wavelength (ZDW) in the 1.3-1.8 $\mu \mathrm{m}$ range. For example, when the core distance is $2.4 \mu \mathrm{m}$, the ZDW of the antisymmetric TE mode is located at $1.65 \mu \mathrm{m}$. If the core-to-core distance reduces down to 2.2 $\mu \mathrm{m}$, the ZDW of the antisymmetric TE mode shifts to $1.73 \mu \mathrm{m}$. In other words, moving the ZDW of the antisymmetric TE mode by $1 \mathrm{~nm}$ requires displacing the two cores by $\sim 2.5 \mathrm{~nm}$ relative to each other. If a mechanism is introduced into the fiber structure to move one of the 
two cores, e.g., by opening a slot on the side of one core in the fiber such that air pressure can be applied on the open membrane [12], continuous dispersion tuning can be realized.

The sensitivity of such dispersion tunability to the core-to-core distance can be further enhanced when the ZDW of the TE or TM mode is located where the slope of the ZDW versus core-to-core distance curve is high (see Fig.9(b)). With a proper design of the fiber structure, this sort of dispersion tunability is interesting and could be useful, for example for fast switching the fiber dispersion from the anomalous dispersion region to the normal dispersion region, in particular in the $1.55 \mu \mathrm{m}$ telecommunication window e.g. for tunable dispersion compensation. Other applications could be in the control of nonlinear optical processes, e.g. for tuning phasematching conditions for four-wave mixing-based frequency conversion [25] or for tailoring of supercontinuum generation from femtosecond laser pulses [26].
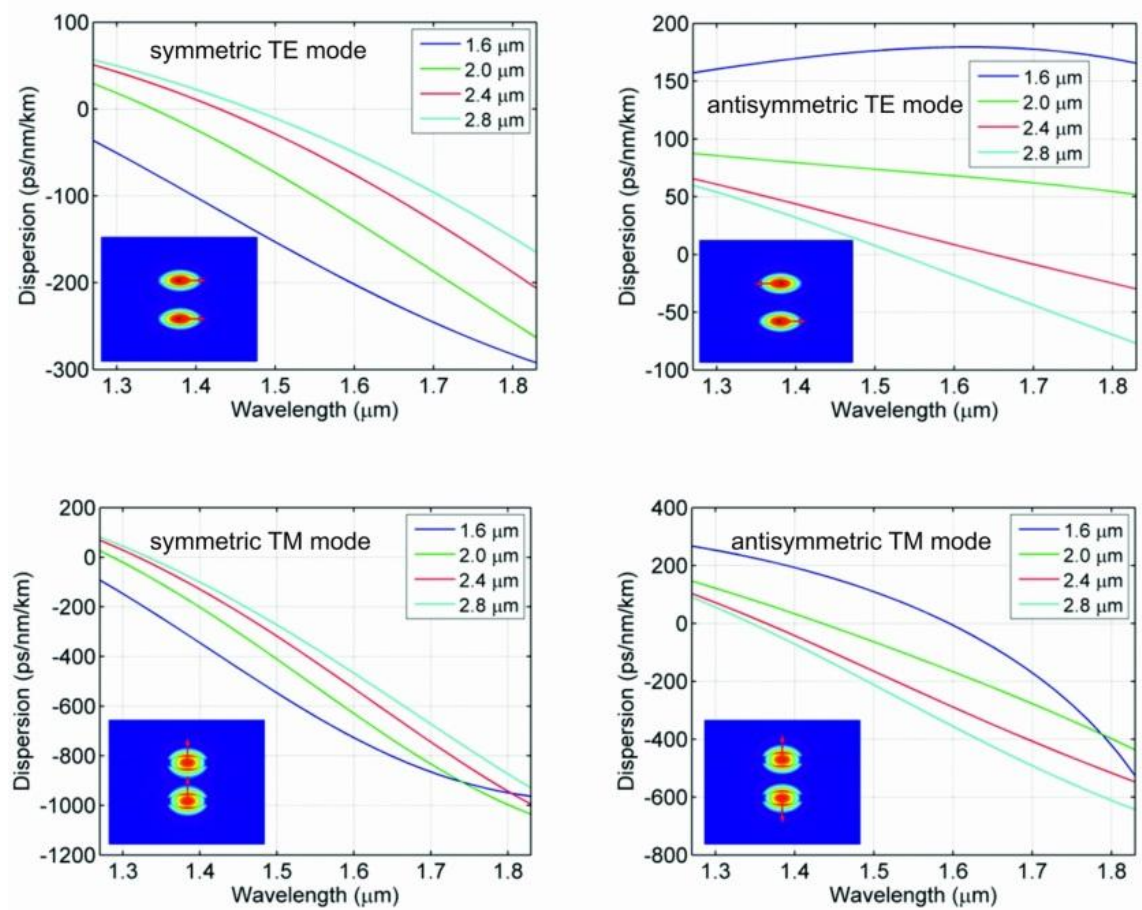

(a)

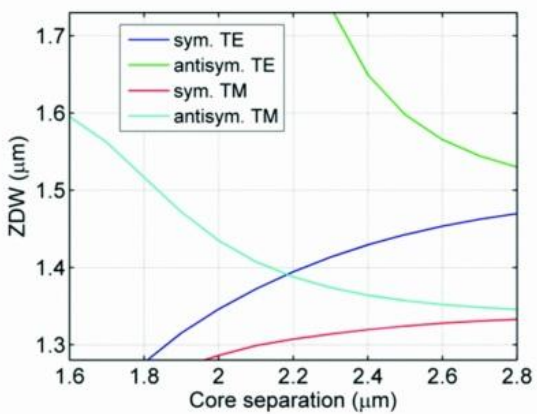

(b)

Fig. 9. Dispersion tunability of dual-core fiber (cores dimension: 2.7 x $0.78 \mu \mathrm{m}$ ). (a) Dispersion profiles of coupled supermodes (see insets for mode functions) for core-to-core separations of 1.6-2.8 $\mu \mathrm{m}$. (b) Zero dispersion wavelength versus core separation for these modes.

\subsection{Connecting of dual-ASC fiber to all-solid silica fiber by arc fusion splicing}


For practical purpose, it is important to able to integrate the multiple parallel ASC fibers with conventional silica fiber. In comparison with other alternatives like connectors and free space coupling, splicing (mechanical splicing and fusion splicing) is a key technology to achieve compact and robust all-fiber devices. In this work, a commercial fusion splicer (Model: Ericsson FSU 975) was employed to splice a commercial silica fiber with the dual-ASC HF shown in Fig. 4. An asymmetric arc-fusion splicing configuration [20, 27], i.e., moving the heating element along the silica fiber from the gap (see Fig. 10(a)), was adopted to splice the borosilicate dualASC fiber with a commercial high-NA small core silica fiber (Nufern UHNA4, NA=0.35). This asymmetric configuration heats but does not significantly soften the silica fiber, while heating the borosilicate glass fiber to above its softening temperature directly via the heating elements and indirectly via the silica fiber. Thus, the temperature at the end of the silica fiber is greater than the temperature at the end of the dual-ASC fiber. This temperature gradient serves to improve thermal diffusion between the two fibers when brought into contact thereby strengthening the fusion splice. The spliced fiber was then protected with a heat-shrink sleeve (see Fig.10(b)).

The lowest splice loss of $2.7 \mathrm{~dB} /$ joint has been achieved at $1550 \mathrm{~nm}$. The calculated mode field area mismatch between the symmetric fundamental TE mode of the dual-ASC fiber and the commercial Nufern silica fiber (MFD: $4 \pm 0.3 \mu \mathrm{m}$ at $1550 \mathrm{~nm}$ ) causes $3.3 \mathrm{~dB}$ loss. The deformation of the holey cladding of the dual-ASC fiber on the spliced segment could cause some increase of the mode area of the dual-ASC fiber and lead into the extra improvement of the splice loss.

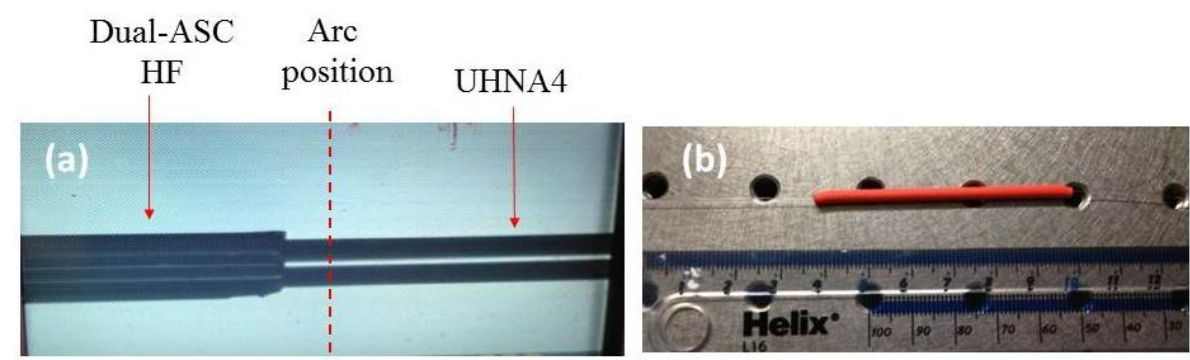

Fig. 10. (a) Photograph of splicing dual-ASC fiber with UHNA4 fiber and (b) spliced fiber with protector sleeve.

\section{Conclusions}

In summary, we have successfully demonstrated a novel sheet-stacking technique to fabricate multiple parallel air-suspended-core optical fibers, using borosilicate glasses as the host materials. A dual-ASC fiber and a 10-ASC fiber have been fabricated. Measurements show that the loss of the fabricated multiple parallel ASC fiber can be as low as the loss of the bulk material. Such a new fabrication method shows advantages in further increasing the number of suspended cores over what is possible with alternative glass extrusion and capillary stacking methods, in terms of the extra loss introduced by the fabrication approach and the uniformity of the membrane structure. In addition, the core material can be different from the supporting membrane material as long as they are thermally compatible. Numerical simulations show that this multiple ASC fiber can be used as a dispersive fiber component with continuously tunable dispersion. Finally, arc fusion splicing has been successfully demonstrated for connecting a dual- 
ASC fiber with a conventional small-core silica fiber, indicating the feasibility of integrating the multiple ASC fiber into existing silica optical fiber systems.

\section{Acknowledgments}

This work was supported by the UK Engineering and Physical Sciences Research Council (EPSRC), under the EPSRC Centre for Innovative Manufacturing in Photonics, the "Photonics Hyperhighway" Programme Grant, and the "Continuously tunable optical buffer" grant (EP/J012874/1).

\section{References}

[1] T. Morioka, New generation optical infrastructure technologies: EXAT initiative towards 2020 and beyond, OECC 2009 (13-17 July 2009 Hong Kong), FT4.

[2] T. Hayashi, T. Taru, O. Shimakawa, T. Sasaki, E. Sasaoka, Design and fabrication of ultra-low crosstalk and low-loss multi-core fiber, Opt. Express, 19 (2011) 16576-16592.

[3] R. Hereth and G. Schiffner, Broad-band optical directional couplers and polarization splitter, J. Lightwave Technol. 7 (1989) 925-930.

[4] P. Pavel and J. Kanka, Erbium-doped twin-core fiber narrow-band filter for fiber lasers, Opt. and Quant. Electron. 33 (2001) 571-581.

[5] D. Gauden, D. Mechin, C. Vaudry, P. Yvernault, D. Pureur, Variable optical attenuator based on thermally tuned Mach-Zehnder interferometer within a twin core fiber, Opt. Commun. 231 (2004) 213-216.

[6] P.M. Blanchard, J.G. Burnett, G.R.G. Erry, A.H. Greenaway, P. Harrison, B. Mangan, J.C. Knight, P.St.J. Russell, M.J. Gander, R. McBride, J.D.C. Jones, Two-dimensional bend sensing with a single, multi-core optical fibre , Smart Mater. Struct. 9 (2000) 132-140.

[7] P.K. Cheo, A. Liu, G.G. King, A High-Brightness laser Beam from a phase-locked multicore Yb-doped fiber laser array, IEEE Photon. Technol. Lett., 13 (2001) 439-441.

[8] Y.M. Huo, P.K. Cheo, G.G. King, Fundamental mode operation of a 19-core phase-locked Yb-doped fiber amplifier, Opt. Express, 12 (2004) 6230-6239.

[9] J. Gao, X. Zhang, L. Shi, W. Shi; Y. Huang, X. Ren, Dispersion characteristics analysis of asymmetric multicore fibers, Proc. SPIE 8307, Passive Components and Fiber-Based Devices VIII, 830720

[10] P. Kaiser, E.A.J. Marcatili, S.E. Miller, A New Optical Fiber, The Bell System Technical Journal 52 (1973) 265-569.

[11] Z. Lian, X. Feng, P. Horak, L. Xiao, Y. Jeong, N. White, K. Frampton, J. Tucknott, H. Rutt, D.N. Payne, W. Stewart, W.H. Loh, Optical fiber with dual cores suspended in air, ECOC 2011 (18-22 Sept. 2011, Geneva, Switzerland), Mo.2.LeCervin.1.

[12] Z. Lian, P. Horak, X. Feng, L. Xiao, K. Frampton, N. White, J.A. Tucknott, H. Rutt, D.N. Payne, W. Stewart, W.H. Loh, Nanomechanical optical fiber, Opt. Express 20 (2012) 29386-29394.

[13] N. Podoliak, Z. Lian, W. H. Loh, and P. Horak, Design of dual-core optical fibers with NEMS functionality, Opt. Express (2014) 1065-1076.

[14] M.L. Povinelli, M. Loncar, M. Ibanescu, E.J. Smythe, S.G. Johnson, F. Capasso, J.D. Joannopoulos, Evanescent-wave bonding between optical waveguides, Opt. Lett. 30 (2005) 3042-3044. 
[15] M. Li, W.H.P. Pernice, C. Xiong, T. Baehr-Jones, M. Hochberg, H.X. Tang, Harnessing optical forces in integrated photonic circuits, Nature 456 (2008) 480-484.

[16] A. Butsch, M.S. Kang, T.G. Euser, J.R. Koehler, S. Rammler, R. Keding, P.St.J. Russell, Optomechanical nonlinearity in dual-nanoweb structure suspended inside capillary fiber, Phys. Rev. Lett. 109 (2012) 183904.

[17] A. Butsch, Optomechanical interactions in microstructured silica fibers, Doctoral Thesis, Friedrich-AlexanderUniversität $\quad$ Erlangen-Nürnberg $\quad$ (FAU), $\quad$ 03.08.2012. http://opus4.kobv.de/opus4fau/frontdoor/index/index/docId/2325.

[18] N.Y. Joly, T.A. Birks, A. Yulin, J.C. Knight, and P.St.J. Russell, Linear and nonlinear guidance in an ultralow loss planar glass membrane, Opt. Lett. 30 (2005) 2469-2471.

[19] K.M. Kiang, K. Frampton, T.M. Monro, R. Moore, J. Tucknott, D.W. Hewak, D.J. Richardson, H.N. Rutt, Extruded singlemode non-silica glass holey optical fibres, Electron. Lett. 38 (2002) 546-547.

[20] X. Feng, F. Poletti, A. Camerlingo, F. Parmigiani, P. Petropoulos, P. Horak, G.M. Ponzo, M. Petrovich, J. Shi, W.H. Loh, D.J. Richardson, Dispersion controlled highly nonlinear fibers for all optical processing at telecoms wavelengths, Opt. Fiber Technology 16 (2010) 378-39.

[21] J. Shi, X. Feng, Z. Lian, N.M. White, P. Horak, W. Loh, A Sheet-Stacking Technique for Making Multiple AirSuspended-Core Optical Fibres, in 3rd Workshop on Specialty Optical Fiber and their Applications (WSOF) (28-30, Aug. 2013, Sigtuna, Sweden), T1.2.

[22] P. Kaiser, H.W. Astle, Low-loss single-material fibers made from pure fused silica, Bell System Technical Journal, 53 (1974) 1021-1039.

[23] Schott

glass catalogue

online:

http://www.schott.com/advanced_optics/us/abbe_datasheets/schott_datasheet_all_us.pdf.

[24] T. Pertsch, T. Zentgraf, U. Peschel, A. Bräuer, F. Lederer, Beam steering in waveguide arrays, Appl. Phys. Lett. 80 (2002) 3247-3249.

[25] P. Dupriez, F. Poletti, P. Horak, M. N. Petrovich, Y. Jeong, J. Nilsson, D. J. Richardson, and D. N. Payne, Efficient white light generation in secondary cores of holey fibers, Opt. Express 15 (2007) 3729-3736.

[26] M.-L. V. Tse, P. Horak, F. Poletti, N. G. Broderick, J. H. Price, J. R. Hayes, and D. J. Richardson, Supercontinuum generation at $1.06 \mu \mathrm{m}$ in holey fibers with dispersion flattened profiles, Opt. Express 14 (2006) 4445-51.

[27] S. Jiang, J. Wang, Method of fusion splicing silica fiber with low-temperature multi-component glass fiber, US Patent 6705771. 
We demonstrate the fabrication of a novel type of optical fibers with multiple parallel airsuspended cores by the sheet-stacking method.

No extra scattering loss from the fabrication process was observed in a fabricated dual airsuspended core fiber.

We propose to use the multiple parallel suspended-core specialty optical fibers as an optical fiber device for tuning dispersion.

Fusion splicing has been successfully used to connect such a multiple suspended core fiber with a conventional silica fiber. 